\title{
Real-time and Accurate Acoustic Indoor Localization With a Smartphone
}

\author{
Xiangji Wen ${ }^{\mathrm{a}}$, Danjie Huang ${ }^{\mathrm{b}}$, Wenhao Fang ${ }^{\mathrm{c}}$, \\ Feng Lin ${ }^{d}$, Yifan Huang ${ }^{e}$, Zhi Wang ${ }^{\dagger}$
}

School of Control Science and Engineering, Zhejiang University,Hangzhou, 310027, China

aemail: zju_wxj@163.com, bemail:huangdanjie07@zju.edu.cn, cemail:wh_fang@zju.edu.cn, demail:21532066@zju.edu.cn, eemail:21532124@zju.edu.cn, femail:wangzhizju@gmail.com

Keywords: LFM; Robust; Real-time performance; Accurate

\begin{abstract}
The requirement for specific indoor location is more and more pressing. However, traditional approaches can only achieve a low accuracy. In this paper, we put forward a new approach to evaluate the target position with the acoustical Linear Frequency Modulation Signal(LFM). Comparing with other acoustical approaches, it is robust to noise, and most importantly it can realize a high accuracy and a good real-time performance. Besides that, several innovative location methods are also compared and verified to promote the real-time, accurate and robust performance.
\end{abstract}

\section{Introduction}

With the rapid development of science and technology, indoor positioning technique is becoming more and more important, for example, mobile robot localization, intelligent conference, garage localization, store localization and so on [1][2][3]. Because of the fact that Global Position System(GPS) can only be used in outdoor places [4][5][6], other techniques also need to be researched to realize the goal of accurate indoor localization. Montaser proposed a low cost approach with Radio Frequency Identification (RFID) technology [7]. The method utilizing WIFI and the approach taking advantage of FM signal strength fingerprinting are also compared. Some other approaches are also summarized by Lymberopoulos [8]. However, the main drawback of these approaches is low accurate [9].

Kaikai Liu use high-band acoustic signals to evaluate the position of the target [10]. However, he utilizes simple-frequency signal so as to be susceptible to the environment. Besides, this system needs lots of beacons and can't achieve a good real-time performance, which brings some inconvenience. Peng Chunyi takes advantage of Beep-Beep algorithm to realize a high accuracy of distance evaluation[11]. However, he don't do some research on the location evaluation field. It requires a good real-time performance to track the target.

Combined with the Beep-Beep, we have done some experiment on the acoustic signal selection. The sound signal selection process is most essential to prove its immunity to noise and improve the accuracy. By evaluating the distance of two nodes or their clock difference, we could estimate the target position with many kinds of location algorithm, for example, TDAO, TOA, DOA and so on, and this will be talked in detail later. The accuracy and real-time performance of different algorithm will also be analyzed and compared. According to the scene, we design a new algorithm to improve both the accuracy and real-time performance successfully.

The flow chart of the system is shown as in Fig.1. Firstly, we should select the proper acoustic signal to improve the accuracy of the arrival time detection of the acoustic signal.

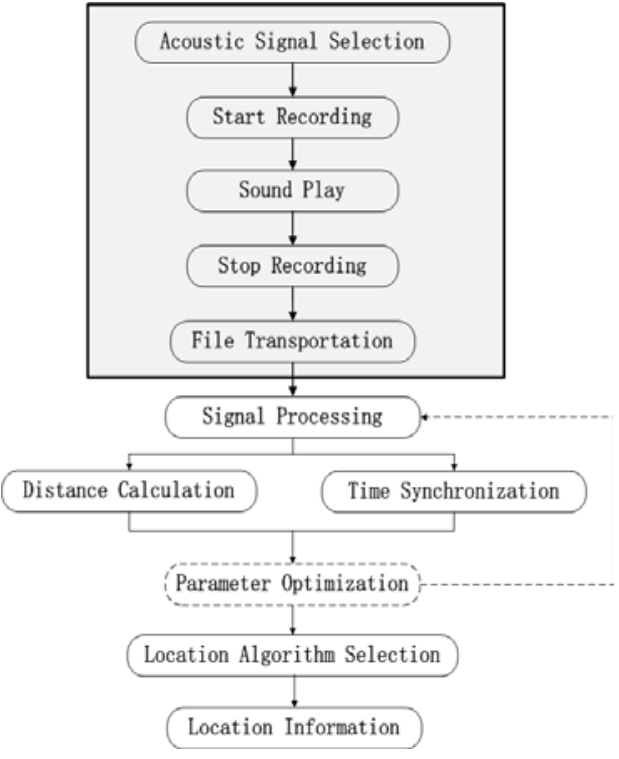

Fig.1. System flowchart. 
Secondly, the target send command to beacons to make them start recording, play sound, stop recording and transport the file to the server as in the gray area in Fig.1. Thirdly, the server will detect the arrival time of acoustic signal to evaluate the distance between two nodes or their time difference. "Parameter Optimization" in the imaginary line is aimed to detect the arrival time of acoustic signal more accurately. At last, we could take advantage of several algorithms to evaluate the target position.

The main contributions of our work are shown as follows:

1) The proper LFM signal is demonstrated to estimate the position of the source with frequency response statistic.

2) We combine the generalized cross correction with LFM signal to achieve a good performance of noise suppression.

3) The weak signal detection method is designed to decrease the number of beacon to most extent.

4) Most excellent feature is verified with high accuracy and good real-time performance with different location methods comparison.

\section{Distance Evaluation Theory and LFM Signal Selection}

In this section, we mainly introduce the process of the theory of distance evaluation and the acoustic signal selection.

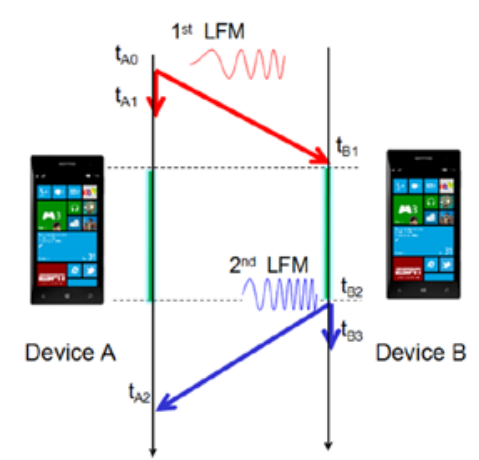

Fig.2. Distance evaluation theory

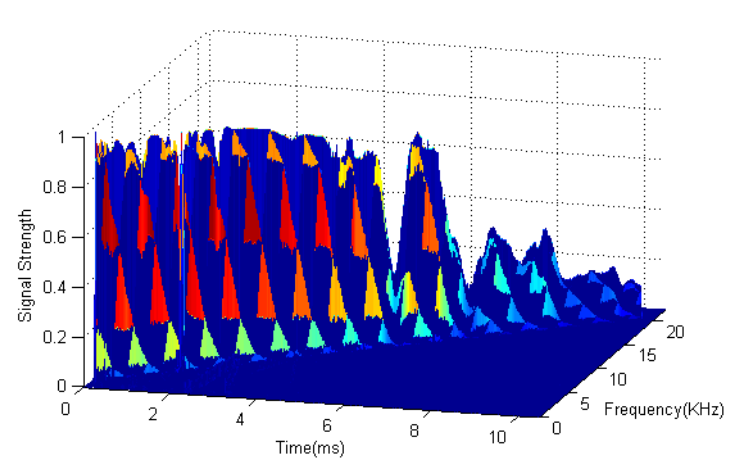

Fig.3. The result of different frequency response.

The theory of distance evaluation is shown as in Fig.2. Firstly, device A and device B open their microphones. Secondly, device A plays the LFM signal. Thirdly, device B also plays the LFM signal after receiving the LFM signal device A plays. At last, when device A received the LFM signal device $\mathrm{B}$ plays, they all close their microphones.

The distance of device A and device B can be calculated by the equations as follows,

$$
\begin{aligned}
& t_{B 1}-\Delta t=t_{A 0}+d / c_{\text {air }} \\
& t_{B 2}-\Delta t=t_{A 2}-d / c_{\text {air }} \\
& c_{\text {air }}=331.45 \sqrt{1+T / 273.15}
\end{aligned}
$$

$\Delta t$ represents their clock offset, $\mathrm{d}$ represents the distance between them, $\mathrm{c}_{\text {air }}$ represents the speed sound spreads, $T$ represents the experimental temperature. According to the fact that the difference between $t_{\mathrm{A} 0}$ and $\mathrm{t}_{\mathrm{A} 0}$ is very little and the same with $\mathrm{t}_{\mathrm{B} 2}$ and $\mathrm{t}_{\mathrm{B} 3}$, the value of $\mathrm{d}$ and $\Delta t$ can be calculated as follows,

$$
\begin{aligned}
& d=\left[\left(t_{A 2}-t_{A 1}\right)-\left(t_{B 3}-t_{B 1}\right)\right] / 2 * c_{\text {air }} \\
& \Delta t=\left[\left(t_{B 1}+t_{B 3}\right)-\left(t_{A 1}+t_{A 2}\right)\right] / 2
\end{aligned}
$$

As mentioned in the introduction, many approaches, are low accurate, for example, RFID, WIFI, signal strength fingerprinting. Different with them, we detect the arrival time of acoustic signal of the target to estimate the distance between them. However, the microphone and speaker of the smartphone have general characteristics and achieve a poor performance in high frequency band. 
We need to select proper acoustic signal to increase its response. Besides, we also select LFM signal to obtain a good anti-interference performance.

In this paper, we design an experiment to measure the different frequency response of the microphone of LG E960 smartphone and optimize the length of LFM signal. The result of the different frequency response is shown as in Fig.3. Because of the fact that the signal intensity decreases rapidly when the frequency is above $10 \mathrm{KHz}$, we select the LFM signal from $3 \mathrm{KHz}$ to $8 \mathrm{KHz}$ as the optical scope.

\section{The Process of The Arrival Time Detection of Acoustic Signal}

In this paper, we take advantage of Generalized cross correlation (GCC) to detect the arrival time of LFM signal. GCC is a very effective method to process given acoustic signal. It has a perfect performance in terms of immunity for disturbance, and most importantly it can achieve a high accuracy with small an amount of calculation.

The original data and the processing result with GCC are shown in Fig.4. It is obvious that the original data contains big noise, and the GCC approach achieves a good performance. Another problem can also be seen in the second subfigure. There are two partial peaks, and the first one is related to the aimed LFM signal and the second one is formulated because of multipath effect. Sometimes the second peak is higher than the first one. According to this concern, we regard the point as the arrival time of regulated signal, whose correlation value is 25 percent of the max one. As shown in Fig.5, the approach can achieve a high accuracy and avoid the multipath effect. The mean value of evaluated distance is nearly equal with the real distance, and the standard value is small, which represents the fact that the evaluated values are centralized distributed.
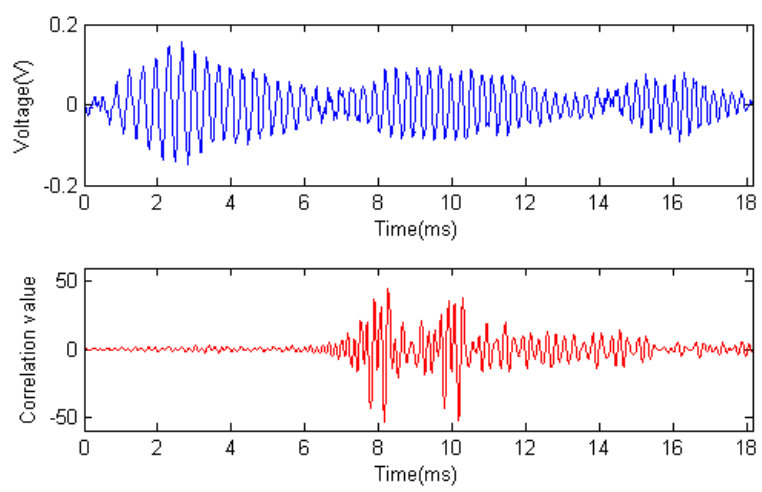

Fig.4. The processing result.
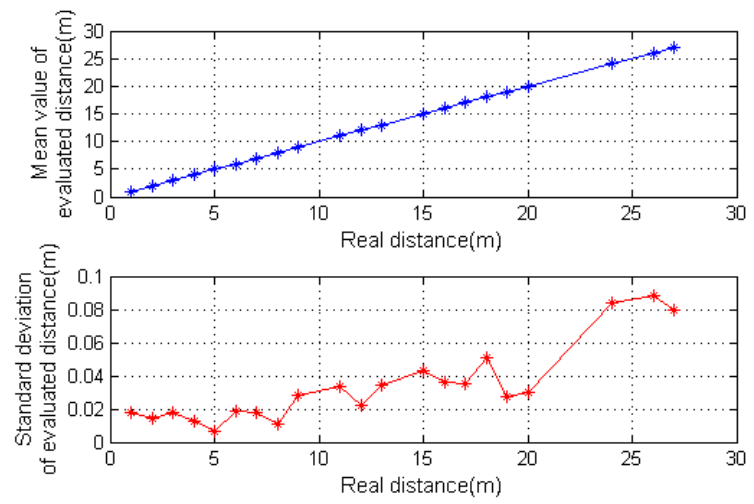

Fig.5. Accuracy over different distances.

\section{The Optimization of Acoustic LFM Signal Length}

It is obvious that the length of the LFM signal is closely related to the immunity from the interference. As the length of the LFM signal increases, the real-time performance will be poor. Therefore, we need to do some research on the field of the evaluated accuracy with different length of the LFM signal, which is demonstrated in Fig.6 and Fig.7. The accuracy and effective proportion decrease as the length become shorter.

The accuracy of estimated distance is evaluated by limited arithmetic mean value $\left(\sigma_{\text {limit }}\right)$, which can be obtained as follows:

Standard Deviation: $\sigma=\sqrt{\sum x^{2} /(n-1)}$,

Arithmetic Mean Value: $\sigma_{\bar{x}}=\sigma / \sqrt{n}$,

Limited Arithmetic Mean Value $: \sigma_{\text {limit }}= \pm t_{a} * \sigma_{\bar{x}}$,

$t_{a}=1.98$ (Confidence Coefficient)

The effectiveness proportion is added up by the $3 \sigma$ principle. In the case of some measurements, according to Gaussian Distribution principle, if all of them only contain random error, the proportion of samples will not exceed 0.3 percent, which are not fallen in the scope ranging from 
$x-3 \sigma$ to $x+3 \sigma$. In our experiment, if we find a sample which is not fallen in this scope, we will regard this sample as illegal one.

According to the statistical result, when the length of LFM signal is over 30ms, the effectiveness proportion can be above 80 percent. However, when the length of the LFM signal is shorter than $30 \mathrm{~ms}$, the effectiveness proportion decreases and the error increases apparently. Combined with the accuracy of different LFM signals, we selected the LFM signal ranging from $3 \mathrm{KHz}$ to $8 \mathrm{KHz}$ as the perfect one with a time window of $30 \mathrm{~ms}$.
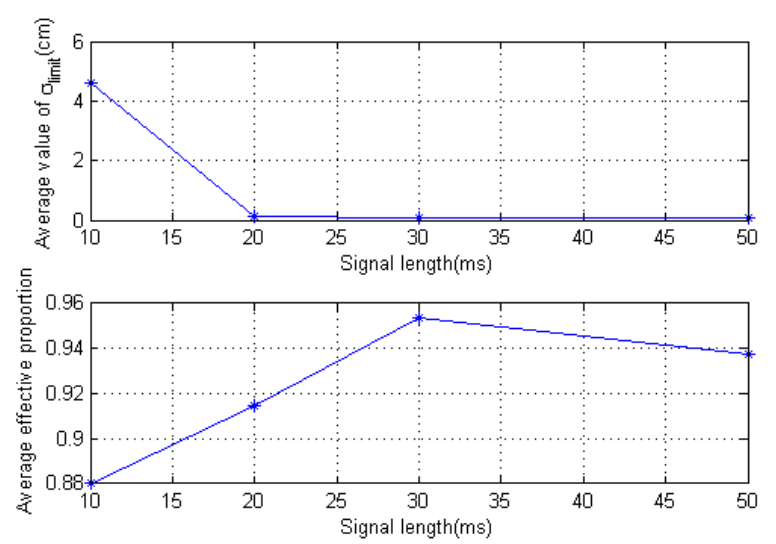

Fig.7. Experiment result over different signal lengths.
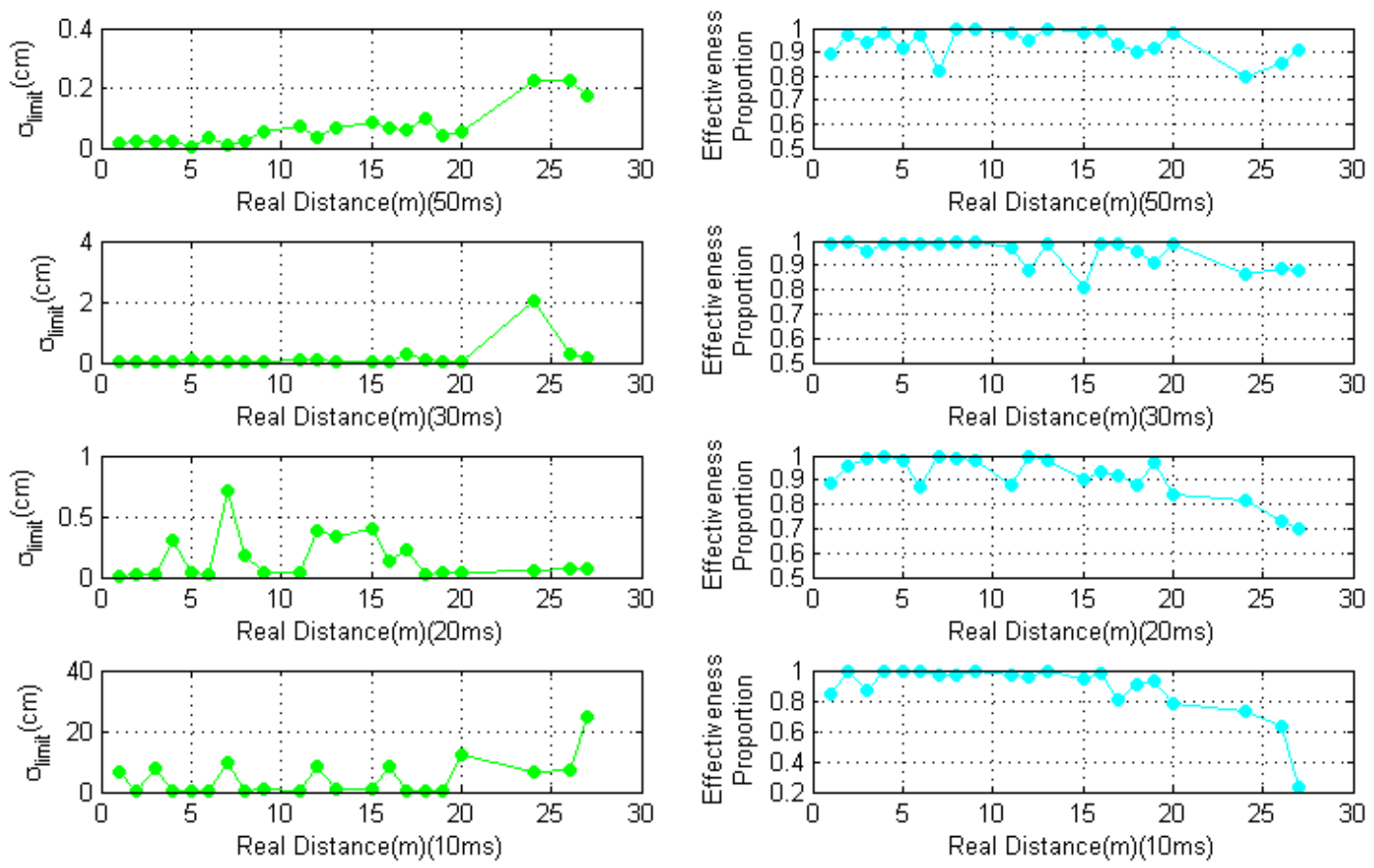

Fig.6. The accuracy and effective proportion over different signal lengths.

\section{Design of Location Schemes}

In this section, we aim to evaluate the geographical coordinate of the target. According to Fig.6, we can conclude that $\sigma_{\text {limit }}$ also increases as the distance increases in general and the distribution of evaluated result above $20 \mathrm{~m}$ will have an apparent difference with the ones below $10 \mathrm{~m}$. In order to reduce the number of beacons and achieve a high accuracy, all of the three experiments are arranged as Fig.8.

With different requirement consideration, for example, high accuracy, good real-time performance or a small amount of beacons, we design three experiments to evaluate the position. They are named as "CBHA", "HRP" and "HRPHA" respectively.

"CBHA" is the abbreviation of "Clumsy But High Accuracy". The

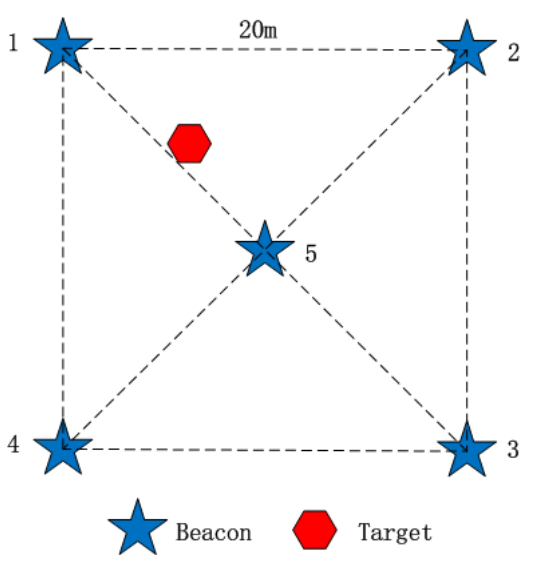

Fig.8. Experiment layout goal of "CBHA" is to obtain a high accurate evaluated result with a small amount of beacons. As we all know, compared with other approaches, if we could learn all the distances between the target and 
all beacons, we could obtain the most accurate evaluated result. Therefore, all the distances can be obtained by taking advantage of a clumsy approach that the target send command to beacons to make them play LFM signal in turn and then play its LFM signal at last. All the distances between the target and the beacons are symbolized by $\mathrm{d}_{1}, \mathrm{~d}_{2}, \mathrm{~d}_{3}, \mathrm{~d}_{4}, \mathrm{~d}_{5}$.

"HRP" is the abbreviation of "High Real-time Performance". The goal of "HRP" is to obtain a high real-time performance. In order to achieve the goal, we take advantage of TDOA positioning principle. The target sends command to the fifth beacon to make them play LFM signal and then play its LFM sgnal at last. The first sound is aimed to create a system clock as follows,

$$
\text { time }_{i 5}=t_{\text {Beaconi }}-t_{\text {Beacon5 }}-10 \sqrt{2} / c_{\text {air }}
$$

$t_{\text {Beacon1 }}, t_{\text {Beacon2 }}, t_{\text {Beacon3 }}, t_{\text {Beacon4 }}$ and $t_{\text {Beacon5 }}$ represents the different arrival times to the beacons of the sound the fifth beacon plays.

The arrival time difference can be detected with equation 5.

$$
\begin{gathered}
\Delta t_{\mathrm{i} 5}=t_{T \text { argeti }}-t_{\text {Target } 5}-\text { time }_{i 5} \\
d_{i}=d_{5}+c_{\text {air }} * \Delta t_{i 5}
\end{gathered}
$$

$\mathrm{t}_{\text {Target } 1}, \mathrm{t}_{\text {Target } 2}, \mathrm{t}_{\text {Target } 3}, \mathrm{t}_{\text {Target} 4}$ and $\mathrm{t}_{\text {Target5 }}$ represents the different arrival times to the beacons of the sound the target plays.

Based on the values of $\Delta \mathrm{t}_{15}, \Delta \mathrm{t}_{25}, \Delta \mathrm{t}_{35}, \Delta \mathrm{t}_{45}$, we could evaluate the coordinate of the target with traditional TDOA approach.

"HRPHA" is the abbreviation of "High Real-time Performance and High Accuracy". It aims to realize a high real-time performance and accurate result. The experimental procedure is the same with "HRP", and the only difference is the process of data processing. After obtaining the values of $\Delta \mathrm{t}_{15}, \Delta \mathrm{t}_{25}, \Delta \mathrm{t}_{35}, \Delta \mathrm{t}_{45}$, we could also calculate the value of $\mathrm{d}_{5}$. Obviously, we could calculate the distances between the target and all the beacons as equation 6 .

Therefore, we could learn the coordinate of the target with a relatively high precision. It is noteworthy that the distance between the target and the fifth beacon should be accurate, which can be realized as shown in Fig.6.

\section{Experiment Arrangement and Experiment Result}

As described in last section, we conduct the experiment in the school playground of Zhejiang University. Firstly, we placed the target casually, and then measured the distances between the target and all beacons with a dedicated a rangefinder. Secondly, we calculated the position of the target with least square algorithm. Thirdly, we sent commands to all the beacons and the target to make them play sound in turn for five times, and then we could get the "CBHA" result. At last, we send command to the target and the fifth beacon to make them play sound in turn, and then we could get the results of "HRP" and "HRPHA" with least square algorithm. The results of all the three algorithms are shown in Fig.9 and Table.1.

The first subgraph represents the evaluated result of "CBHA", the second subgraph represents the evaluated result of "HRP" and the third subgraph represents the evaluated result of "HRPHA". The max error, max error and standard deviation are all similar with each other. The "CBHA" algorithm needs three beacons work at least to evaluate the

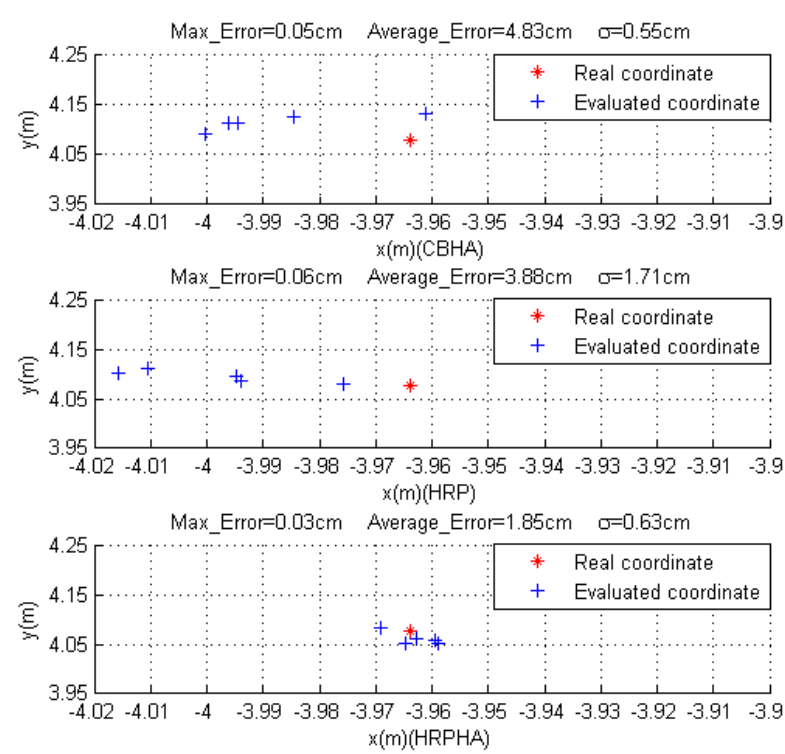

Fig.9. Experiment result.

Table.1. Experiment result.

\begin{tabular}{|c|c|c|c|}
\hline & $\bar{x}_{\text {error }}(\mathrm{cm})$ & $\sigma_{\text {error }}(\mathrm{cm})$ & $\begin{array}{c}\text { Real-time } \\
\text { performance }\end{array}$ \\
\hline CBHA & 4.83 & 0.55 & $780 \mathrm{~ms}$ \\
\hline HRP & 3.88 & 1.71 & $260 \mathrm{~ms}$ \\
\hline HRPHA & 1.85 & 0.63 & $260 \mathrm{~ms}$ \\
\hline
\end{tabular}


position of the target, and all the beacons can't influence each other, which achieve a good robust performance. However, it has a bad real-time performance. The "HRP" algorithm needs four beacons work at least, and it can also achieve a bad robust performance. The "HRPHA" can estimate the position of the target only when three beacons work including the fifth one, which can be achieved at most situation. As described in table 1, it can achieve a high accurate result than "HRP". $\bar{x}_{\text {error }}$ represents the mean value of the evaluated result error except for the abnormal data. $\sigma_{\text {error }}$ represents the standard deviation of the evaluated result error. The most surprising characters are its real-time performance and good robust performance.

\section{Conclusion}

Taking advantage of the LFM signal with a 30ms length, we succeed to estimate the position of the target with a max error of $10 \mathrm{~cm}$. Besides, it achieves a perfect real-time performance, and the "HRPHA" approach is more robust, which can be effective even when there are only three beacons working. The system proposed in this paper will have a high commercial value.

\section{References}

[1] Kim U H, Mizumoto T, Ogata T, et al. Improvement of speaker localization by considering multipath interference of sound wave for binaural robot audition[C]. Intelligent Robots and Systems (IROS), 2011 IEEE/RSJ International Conference on. IEEE, 2011: 2910-2915.

[2] Sur S, Wei T, Zhang X. Autodirective audio capturing through a synchronized smartphone array[C]. Proceedings of the 12th annual international conference on Mobile systems, applications, and services. ACM, 2014: 28-41.

[3] Bingbing Y, Wenbo R, Bolin Y, et al. An indoor positioning algorithm and its experiment research based on RFID[J]. International journal on smart sensing and intelligent systems, 2014, 7(2): 879-897.

[4] Belinska V, Kluga A, Kluga J. Application of least square method with variable parameters for GPS accuracy improvement[J]. Elektronika ir Elektrotechnika, 2015, 104(8): 109-112.

[5] Dujon A M, Lindstrom R T, Hays G C. The accuracy of Fastloc-GPS locations and implications for animal tracking[J]. Methods in Ecology and Evolution, 2014, 5(11): 1162-1169.

[6] Vickery W M, Dascombe B J, Baker J D, et al. Accuracy and reliability of GPS devices for measurement of sports-specific movement patterns related to cricket, tennis, and field-based team sports[J]. The Journal of Strength \& Conditioning Research, 2014, 28(6): 1697-1705.

[7] Montaser A, Moselhi O. RFID indoor location identification for construction projects[J]. Automation in Construction, 2014, 39: 167-179.

[8] Lymberopoulos D, Liu J, Yang X, et al. A realistic evaluation and comparison of indoor location technologies: experiences and lessons learned[C]. Proceedings of the 14th International Conference on Information Processing in Sensor Networks. ACM, 2015: 178-189.

[9] Moghtadaiee V, Dempster A G. Indoor location fingerprinting using FM radio signals[J]. Broadcasting, IEEE Transactions on, 2014, 60(2): 336-346.

[10] Liu K, Liu X, Li X. Guoguo: Enabling fine-grained indoor localization via smartphone[C]. Proceeding of the 11th annual international conference on Mobile systems, applications, and services. ACM, 2013: 235-248.

[11] Peng C, Shen G, Zhang Y, et al. Beepbeep: a high accuracy acoustic ranging system using cots mobile devices[C]. Proceedings of the 5th international conference on Embe- dded networked sensor systems. ACM, 2007: 1-14. 